\title{
A low coefficient of variation in hepatic triglyceride concentration in an inbred rat strain
}

\author{
Tomoko Shimoda', Shota Hori ${ }^{1}$, Kenta Maegawa', Akari Takeuchi ${ }^{1}$, Yeonmi Lee ${ }^{2}$, Ga-Hyun Joe ${ }^{3}$, Yasutake Tanaka ${ }^{4}$,
} Hidehisa Shimizu ${ }^{5}$ and Satoshi Ishizuka ${ }^{1 *}$ (D)

\begin{abstract}
Background: Inbred strains are characterized by less genetic variation, which suggests usefulness of inbred strains for evaluations of various parameters. In this study, experimental reproducibility in several parameters was compared between an outbred Wistar rat and Wistar King A Hokkaido (WKAH/HkmSlc) rat, the inbred strain that is originated from Wistar rats.

Methods: Difference of variations was investigated in parameters of body compositions and liver functions such as body weight, liver weight, liver triglycerides (TG), liver cholesterol and plasma alanine aminotransferase activity (ALT) between WKAH rats and outbred Wistar rats by using the coefficient of variation (CV).

Results: There was no difference in the CVs of body weight and relative liver weight between WKAH and Wistar rats. The CVs of body weight and relative liver weight were below $10 \%$ in both WKAH and Wistar rats. The CVs of TG, cholesterol, and ALT in Wistar rats were between 30 and 40\%, whereas those in WKAH rats were between 10 and $25 \%$. A low CV level of TG was observed in WKAH rats compared to that in Wistar rats regardless of the duration of the experimental period in those rat strains.
\end{abstract}

Conclusion: The low CV values in metabolic parameters involved in liver functions in the inbred rats suggested an advantage of using inbred rather than outbred rats for the evaluation of liver lipid metabolism.

Keywords: Coefficient of variation, Cholesterol, Inbred, Liver, Triglycerides

\section{Background}

In vivo experiments, particularly those using rodents, contribute greatly to understand the pathophysiology of humans. Inbred strains and outbred stocks are the two major classes of laboratory rodents [1]. Inbred strains are generally characterized by genetic uniformity, which results in less phenotypic variation in response to toxic or pharmacologic stimuli compared with outbred stocks [2]. In contrast, outbred stocks are characterized by

\footnotetext{
* Correspondence: zuka@chem.agr.hokudai.ac.jp

${ }^{1}$ Research Faculty of Agriculture, Hokkaido University, Kita-9, Nishi-9, Kita-ku, Sapporo, Hokkaido 060-8589, Japan

Full list of author information is available at the end of the article
}

being genetically variable. This suggests that the use of inbred strains improves experimental reproducibility with fewer animals. Outbred populations differ from randomly bred ones in that they are systematically bred to maintain the maximal genetic heterogeneity [3].

Recently, utilization of inbred strains in research has been proposed based on genetic stability [4], but difference of variation in metabolic parameters was still unclear among inbred and outbred animals. The use of outbred animals is prevailing in many fields of in vivo research. Considering the advantages of using inbred rodents, it is necessary to investigate variation in the parameters of interest between inbred and outbred rodents [5]. In this 
study, the coefficient of variation $(\mathrm{CV})$ in several sets of experiments was compared in body weight, relative liver weight, and metabolic parameters between outbred Wistar rats and Wistar King A Hokkaido (WKAH) rats, an inbred strain of Wistar rats.

\section{Methods}

\section{Animals}

Three-week-old Wistar male rats (Slc:Wistar, Japan SLC, Inc., Shizuoka, Japan) and three-week-old Wistar King A Hokkaido male rats (WKAH/HkmSlc, Japan SLC) (NBRP Rat No: 0154) were used as an outbred stock and an inbred strain, respectively. They were housed in an air-conditioned room at $22 \pm 2{ }^{\circ} \mathrm{C}$ with $55 \pm 5 \%$ humidity, and a light period from 8:00 to 20:00. The rats were housed individually in wire-bottomed cages and allowed ad libitum access to food and water. They were acclimated to a diet based on the American Institute of Nutrition (AIN)-93G formulation [6]. At the end of the experimental period, the rats were anesthetized with sodium pentobarbital $(50 \mathrm{mg} / \mathrm{kg})$ and exsanguinated by blood withdrawal. The plasma and liver were dissected and stored at $-80^{\circ} \mathrm{C}$ until analysis.

\section{Biochemical parameters}

Alanine aminotransferase (ALT) in the plasma was measured using a transaminase CII-test Wako kit (Wako Pure Chemical Corporation, Osaka, Japan). Liver lipids were extracted using chloroform and methanol $(2: 1, \mathrm{v} / \mathrm{v})$. The extracts were evaporated and dissolved in isopropanol and were used to measure triglyceride (TG) and cholesterol in the liver by using the Triglyceride E-test Wako and Cholesterol E-test Wako (Wako), respectively.

\section{Calculation of CVs}

$\mathrm{CV}$ is the proportion of the standard deviation in the mean value. Several sets of experimental data were used in rats fed with the AIN-93G-based diet. In study I, Wistar rats were maintained for 6 weeks (6 rats per group and 4 groups). In study II, WKAH rats were maintained for 2 weeks ( 6 rats per group and 4 groups). In study III, WKAH rats were maintained for 13 weeks (10-12 rats per group and 4 groups). CVs were calculated from the values such as body weight $(\mathrm{g})$, relative liver weight $(\mathrm{g} / \mathrm{kg}$ body weight), liver TG (mg/g liver), liver cholesterol (mg/g liver) and plasma ALT (IU/L) in every group as mentioned above, which resulted in the number of CVs in each study was four (group) in each parameter $(n=4)$. Those calculated CVs were used for the statistical analysis as follows.

\section{Statistical analysis}

The Tukey-Kramer test was used to compare CVs in each parameter among studies I, II, and III. Pearson correlation analysis were performed between $\mathrm{CV}$ of liver
TG concentration and of liver cholesterol concentration and between $\mathrm{CV}$ of body weight and of relative liver weight. JMP software (ver. 14.0; SAS Institute, Caty, NC, USA) was used for the Tukey-Kramer test and Pearson correlation analysis. A probability of less than 0.05 was considered as significant. To determine an adequate sample size to identify significant differences in hepatic iron concentration, a power analysis was performed using G*Power (version 3.1.9.4) [7] based on the experimental design. Based on an $\alpha$ probability of 0.05 and a power of 0.80 , the effect size was estimated using the results from preliminary studies (unpublished results). The required sample (number of group) size was from three to nine per study.

\section{Results}

The CVs of body weight and relative liver weight were around 5 to $10 \%$, whereas those of biochemical parameters were from 10 to $40 \%$ (Fig. 1). There was no significant difference in the CVs of body weight, liver weight, cholesterol, and ALT among the studies. The CV in TG was significantly lower in the study II and III (WKAH for 2 weeks and 13 weeks) than in the study I (Wistar rats for 6 weeks) (Fig. 1). In other biochemical parameters such as cholesterol and ALT, the CVs in the Wistar rats tended to be a higher than those of WKAH rats regardless of the duration of the experimental period. These results showed that the CVs of body and relative liver weight were below $10 \%$, regardless of the genetic background of the rats.

The CV of liver TG correlated with that of liver cholesterol $\left(R^{2}=0.49, P<0.05\right)$ (Fig. 2a). There was a positive correlation $\left(R^{2}=0.41, P<0.05\right)$ between the CVs of body weight and relative liver weight (Fig. 2b). In WKAH rats,

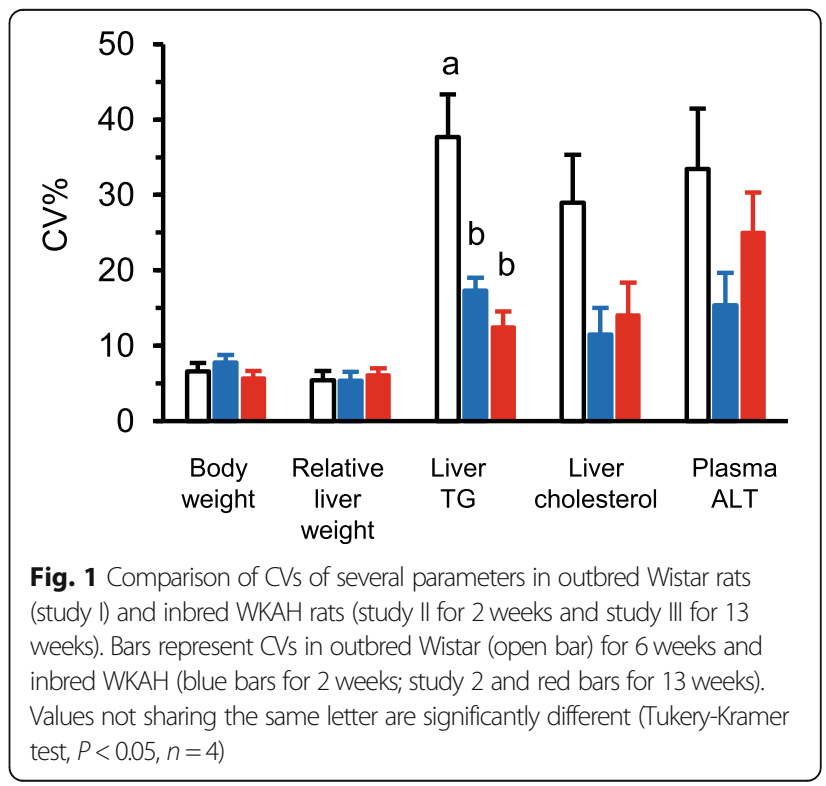


the CV plots of TG and cholesterol were accumulated at relatively low levels (Fig. 2a), whereas those of Wistar rats were scattered at higher levels. Such a trend was not observed in the CVs of body weight and relative liver weight (Fig. 2b).

\section{Discussion}

The CV is frequently used to qualify the accuracy of measurement systems [8]. Since the CV is an indicator of variation, it can evaluate deviation in any aspect of biological parameters. In this study, alteration of CVs was assessed in the weight and metabolism that are frequently used in experimental nutrition research among WKAH (2 weeks and 13 weeks) and Wistar rats. Basically, the CVs of weight (body weight and relative liver weight) were relatively small (below 10\%) in all the rats, whereas those of metabolic parameters such as TG, cholesterol, and ALT were above $10 \%$. Notably, the lower value in the CV of liver TG in the inbred than in outbred rats suggests that the stability of the genetic background in inbred rats avoided the fluctuation in the parameters.

In literature, the CVs of body weight of various inbred mouse strains range from 4.2 to $10.1 \%$ [9]. Similarly, the $\mathrm{CV}$ of body weight in the present study falls within the same range [9] regardless of the genetic background. In the case of metabolic parameters, much higher CVs were reported. For example, CVs in plasma amino acid concentrations were from 3 to $28 \%$ and the majority was around $15 \%$ in clinical samples [10]. The CV of ${ }^{18}$ fluoro2-deoxy-D-glucose incorporation in human pulmonary cancer cells is around $31.9 \%$ [11]. In the case of plasma glucose, the $\mathrm{CV}$ ranges from 34 to $51 \%$ in overnightfasted monkeys [12]. Additionally, a high CV value in $\gamma$ aminobutyric acid concentration makes it difficult to detect the difference between control and treatment groups in the metabolomic analysis of ${ }^{1} \mathrm{H}-\mathrm{NMR}[13]$, indicating the advantage of a lower $\mathrm{CV}$ for detecting differences in metabolite concentrations among treatments.

The prevention of obesity and metabolic disorders is necessary to reduce the risk of noncommunicable diseases [14]. Studies in experimental animals have provided valuable information about biological processes in the development of metabolic diseases, such as obesity, type 2 diabetes, and non-alcoholic fatty liver diseases $[15,16]$, and enabled the establishment of various metabolic markers for the prevention and diagnosis of these disorders. The present study suggests the importance of using inbred animals with lower CV values of metabolites for accurate evaluation of metabolic alterations.

In outbred stocks, it is sometimes difficult to detect a significant difference in biochemical parameters among treatments in toxicology [1]. In contrast, such differences can be detected easily in inbred strains [2] due to stability of genetic background [3]. The CVs in all the parameters in inbred strains are not necessarily less than those in outbred stocks. The present study also showed that the CVs of weight parameters were almost comparable between WKAH and Wistar rats. In nutritional science, rats are preferentially used because their body size enables extraction of sufficient quantities of organs, biological fluids such as blood or intestinal contents, etc. Further, a PubMed search revealed that $80 \%$ of inbred strains used are mice, while $10 \%$ are outbred rats [5]. This observation suggests that researchers do not recognize the advantage of using inbred strains to detect a difference in metabolic parameters among treatments, especially in rat studies.

To obtain a significant difference between the values in biological parameters, CVs of about $7 \%$ in each value are generally required [17]. Since the CVs of body weight and relative liver weight fell in this range (Fig. 1), a change in these body composition parameters can be easily detected even in outbred stocks. In other words, detection of significant difference in metabolic parameters such as TG,
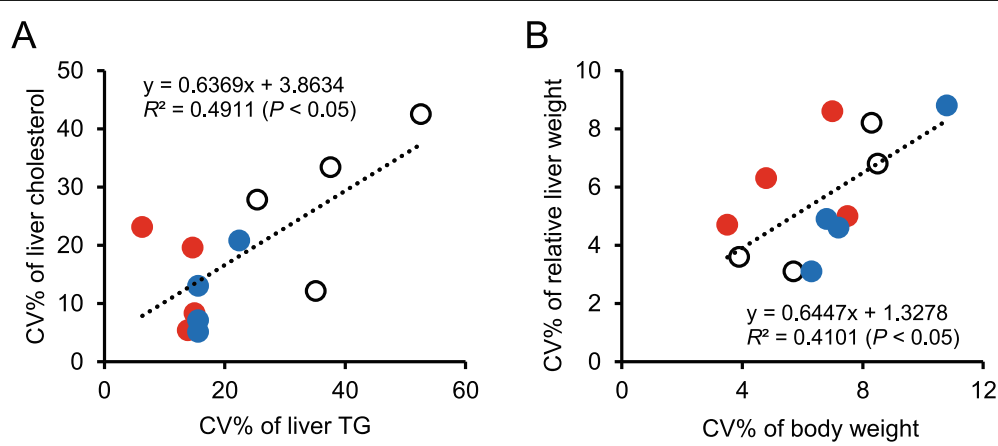

Fig. 2 Pearson correlation between the CVs of parameters determined. a CVs of liver TG and liver cholesterol in outbred Wistar rats (study I) and inbred WKAH rats (study II for 2 weeks and study III for 13 weeks). b CV of body weight and CV of relative liver weight in outbred Wistar rats (study I) and inbred WKAH rats (study II for 2 weeks and study III for 13 weeks). Each spot shows the individual CVs in each group of Wistar rats (open circle, 6 weeks) or WKAH rats (blue circle, 2 weeks; red circle, 13 weeks) 
cholesterol, and ALT, would be difficult, especially in outbred stocks.

This study suggests an advantage of inbred rats to detect differences especially in several metabolic parameters in rats, which contributes to reduction in number of animals in an experiment. It should be noted that an appropriate sample size depends on the parameters of interest.

\section{Conclusion}

The present study demonstrated that the CV of liver TG in inbred WKAH rats was significantly less than that in outbred Wistar rats. The results suggest that the use of inbred rats can help in the accurate evaluation of metabolic parameters in an animal experiment. To find relevant parameters in animal studies is expected to contribute to elucidation of significant connection between parameters in a clinical study although there might be much broader deviations of metabolic parameters in clinical studies.

\section{Abbreviations}

ALT: alanine aminotransferase; CV: coefficient of variation; TG: triglyceride

\section{Acknowledgements}

Not applicable.

\section{Authors' contributions}

SI designed and supervised the implementation of this study; TS, SH, KM, AT, YL, GHJ, YT, and HS performed experiments; TS, and SI analyzed data and wrote the paper; All the authors read and approved the final manuscript.

\section{Funding}

This work was supported by JSPS KAKENHI Grant Numbers 16 K14917, 19H2900 and JST COI Grant Number JPMJCE1301. The authors declare no conflicts of interest.

\section{Availability of data and materials}

The datasets analyzed during the current study are included in the manuscript.

\section{Ethics approval}

The present study was approved by the Institutional Animal Care and Use Committee of National Corporation, Hokkaido University (approval number: 14-0026 and 17-0119), and all animals were maintained in accordance with the Hokkaido University Manual for Implementing Animal Experimentation.

\section{Consent for publication}

Not applicable.

\section{Competing interests}

The authors declare that they have no competing interests.

\footnotetext{
Author details

'Research Faculty of Agriculture, Hokkaido University, Kita-9, Nishi-9, Kita-ku, Sapporo, Hokkaido 060-8589, Japan. ${ }^{2}$ Lee Gil Ya Cancer and Diabetes Institute, Gachon University, 155, Gaetbeol-ro, Yeonsu-gu, Incheon 21999, Korea. ${ }^{3}$ Research Faculty of Fisheries, Hokkaido University, 3-1-1, Minato-cho, Hakodate, Hokkaido 041-8611, Japan. ${ }^{4}$ Faculty of Agriculture, Graduate School, Kyushu University, 744, Motooka, Nishi-ku, Fukuoka, Fukuoka 819-0395, Japan. ${ }^{5}$ Institute of Agricultural and Life Science, Academic Assembly, Shimane University, 1060, Nishikawatsu-cho, Matsue, Shimane 690-8504, Japan
}

Received: 8 May 2020 Accepted: 10 June 2020

Published online: 16 June 2020

References

1. Festing MFW. Variation and its implications for the design of experiments in toxicological research. Comp Haematol Int. 1997;7:202-7.

2. Festing MFW. Inbred strains should replace outbred stocks in toxicology, safety testing, and drug development. Toxicol Pathol. 2010;38:681-90.

3. Gill TJ. The use of randomly bred and genetically defined animals in biomedical research. Am J Pathol. 1980;101:S21-32.

4. Vaickus $\amalg$, Bouchard J, Kim J, Natarajan S, Remick DG. Inbred and outbred mice have equivalent variability in a cockroach allergen-induced model of asthma. Comp Med. 2010;60:420-6.

5. Festing MFW. Evidence should trump intuition by preferring inbred strains to outbred stocks in preclinical research. ILAR J. 2014:55:399-404.

6. Lee Y, Yoshitsugu R, Kikuchi K, Joe GH, Tsuji M, Nose T, Shimizu H, Hara H, Minamida K, Miwa K, Ishizuka S. Combination of soya pulp and Bacillus coagulans lilac-01 improves intestinal bile acid metabolism without impairing the effects of prebiotics in rats fed a cholic acid-supplemented diet. Br J Nutr. 2016;116:603-10.

7. Faul F, Erdfelder E, Lang AG, Buchner A. G*power 3: a flexible statistical power analysis program for the social, behavioral, and biomedical sciences. Behav Res Methods. 2007;39:175-91.

8. Hundal RS, Petersen KF, Mayerson AB, Randhawa PS, Inzucchi S, Shoelson SE, Shulman GI. Mechanism by which high-dose aspirin improves glucose metabolism in type 2 diabetes. J Clin Invest. 2002;109:1321-6.

9. Konarzewski M, Diamond J. Evolution of basal metabolic rate and organ masses in laboratory mice. Evolution. 1995:49:1239-48.

10. Felig P, Owen OE, Wahren J, Cahill GF Jr. Amino acid metabolism during prolonged starvation. J Clin Invest. 1969:48:584-94

11. Nolop KB, Rhodes CG, Brudin LH, Beaney RP, Krausz T, Jones T, Hughes JMB. Glucose utilization in vivo by human pulmonary neoplasms. Cancer. 1987; 60:2682-9.

12. Young AA, Gedulin BR, Bhavsar S, Bodkin N, Jodka C, Hansen B, Denaro M Glucose-lowering and insulin-sensitizing actions of exendin-4: studies in obese diabetic $(o b / o b, d b / d b)$ mice, diabetic fatty Zucker rats, and diabetic rhesus monkeys (Macaca mulatta). Diabetes. 1999:48:1026-34

13. Tkáč I, Keene CD, Pfeuffer J, Low WC, Gruetter R. Metabolic changes in quinolinic acid-lesioned rat striatum detected non-invasively by in vivo ${ }^{1} \mathrm{H}$ NMR spectroscopy. J Neurosci Res. 2001;66:891-8.

14. Alwan A. Global status report on noncommunicable diseases 2010. World Health Organization 2011. https://www.who.int/nmh/publications/ncd_ report2010/en/. Accessed 13 Jun 2020.

15. Kleinert M, Clemmensen C, Hofmann SM, Moore MC, Renner S, Woods SC, Huypens P, Beckers J, de Angelis MH, Schürmann A, Bakhti M, Klingenspor M, Heiman M, Cherrington AD, Ristow M, Lickert H, Wolf E, Havel PJ, Müller TD, Tschöp MH. Animal models of obesity and diabetes mellitus. Nat Rev Endocrinol. 2018:14:140-62.

16. Febbraio MA, Reibe S, Shalapour S, Ooi GJ, Watt MJ, Karin M. Preclinical models for studying NASH-driven HCC: how useful are they? Cell Metab. 2019;29:18-26

17. Kobayashi K, Sakuratani Y, Abe T, Yamazaki K, Nishikawa S, Yamada J, Hirose A, Kamata E, Hayashi M. Influence of coefficient of variation in determining significant difference of quantitative values obtained from 28-day repeateddose toxicity studies in rats. J Toxicol Sci. 2011:36:63-71.

\section{Publisher's Note}

Springer Nature remains neutral with regard to jurisdictional claims in published maps and institutional affiliations. 